\title{
Painleve analysis and exact solutions of two dimensional Korteweg-de Vries-Burgers equation
}

\author{
M P JOY \\ Materials Research Centre, Indian Institute of Science, Bangalore 560012, India \\ MS received 5 April 1995; revised 26 September 1995
}

\begin{abstract}
Two dimensional Korteweg-de Vries-Burgers equation is shown to be non-integrable using Painleve analysis. Exact travelling wave solutions are obtained using an algorithmic approach of truncating the Painleve series expansions.
\end{abstract}

Keywords. Korteweg-de Vries-Burgers equation; Painleve analysis; exact solutions.

PACSNos 02.30; 03.40

\section{Introduction}

Painleve analysis is a powerful tool in investigating the integrability properties of differential equations $[1,2,3]$. It can be used to find Lax pairs, Backlund transformations, Hirota's bilinear equations, symmetries, invariants, etc., of integrable equations. It can also be used to find exact solutions of non-integrable equations in an algorithmic way [4]. In this paper we present the Painleve analysis of two dimensional Kortewegde Vries-Burgers equation (2d-KdVB),

$$
\left(u_{t}+u u_{x}+\mu u_{x x x}-v u_{x x}\right)_{x}+\quad f f u_{y y} \sim 0 .
$$

It is a two-dimensional generalization of KdVB equation which is used as a non-linear wave model of fluid flow in an elastic tube with dispersion and dissipation, flow of liquids containing small bubbles, etc. $[5,6]$. The $2 \mathrm{~d}-\mathrm{KdVB}$ serves as a model for propagation of shallow water waves subject to a small transverse disturbance and influenced by viscosity. Similarity solutions of such systems are discussed in [7]. Recently travelling wave solutions of this system were derived using different methods $[8,9,10]$. In all these methods they assumed solutions with travelling wave form and substituted such a solution in the system and determined the exact parameter values at which they exist, if they exist.

Using the technique of truncating the Painleve series expansions at different orders we obtained exact travelling wave solutions of this equation without assuming any particular form for the solutions a priori. Equation (1) is found to be non-Painlevé type and due to Painleve conjecture it is non-integrable, but it has got conditional Painleve property. When $\mathrm{v},=0$ and $a=0$ it becomes $\mathrm{KdV}$ equation and when $\mathrm{v}=0$ it becomes KP equation. Both of them are integrable and have soliton solutions. When $\mu=0$ and $a=0$ it becomes Burgers equation which is also integrable. At $G=0$ it is $\mathrm{KdVB}$ 
equation and at $\mu=0$ it is $2 \mathrm{~d}$-Burgers equation, both of them are non-integrable. Painleve analysis of KdVB is given in [11].

In the next section we present the Painleve analysis of $2 d-K d V B$. In $\$ 3$ exact solutions of the system are given. Last section summarises the results and conclusion.

\section{Painleve analysis}

In Painleve analysis we expand the solution $u$ about a singular manifold $\phi(x, y, t)=0$ in an infinite series

$$
u=\phi^{\alpha} \sum_{j=0}^{\infty} u_{j} \phi^{j},
$$

where a is a negative integer determined by balancing the powers of $\phi$ of dominant terms in the equation. $\phi$ is a non-characteristic manifold. Coefficients $u_{j}$ are functions of $\mathrm{x}, y$ and $t$. If solutions are single valued about the movable singular manifold, the partial differential equation is said to have Painleve property. There are basically three steps in the Painleve analysis, viz, dominant behaviour analysis, finding the resonances, and checking whether arbitrary coefficients enter at the resonance values [3].

From the dominant behaviour analysis we get $\alpha=-1$. By balancing the terms of order $\phi^{6}{ }^{6}$ in the equation after substituting (2) for $u(x, y, t)$ in (1), we obtain recurrence relations for $u_{j}$

$$
\begin{aligned}
& (j-4)(j-5) u_{j-2} \phi_{x} \phi_{t}+(j-5)\left[u_{j-3} \phi_{x t}+u_{j-3, x} \phi_{t}+u_{j-3, t} \phi_{x}\right] \\
& \quad+u_{j-4, x t}+(j-k-2)(k-2) u_{j-k} u_{k} \phi_{x}^{2}+2(j-k-2) u_{j-k} u_{k-1, x} \phi_{x} \\
& \quad+u_{j-k-1, x} u_{k-1, x}+(k-2)(k-3) u_{j-k} u_{k} \phi_{x}^{2}+(k-3) u_{j-k} u_{k-1} \phi_{x x} \\
& +2(k-3) u_{j-k} u_{k-1, x} \phi_{x}+u_{j-k} u_{k-2, x x}+\mu\left\{(j \sim 2)(j-3)(j-4)(j-5) u_{j} \phi_{x}^{4}\right. \\
& \quad+(j-3)(j-4)(j-5)\left[6 u_{j-1} \phi_{x}^{2} \phi_{x x}+4 u_{j-1, x} \phi_{x}^{3}\right] \\
& +(j-4)(j-5)\left[3 u_{j-2} \phi_{x x}^{2}+4 u_{j-2} \phi_{x} \phi_{x x x}\right. \\
& \left.+12 u_{j-2, x} \phi_{x} \phi_{x x}+6 u_{j-2, x x} \phi_{x}^{2}\right]+(j-5)\left[u_{j-3} \phi_{x x x x}+4 u_{j-3, x} \phi_{x x x}\right. \\
& \left.\left.+6 u_{j-3, x x} \phi_{x x}+4 u_{j-3, x x x} \phi_{x}\right]+u_{j-4, x x x x}\right\} \\
& \quad-v\left\{(j-4)(j-5)\left[(j-3) u_{j-1} \phi_{x}^{3}+3 u_{j-2} \phi_{x} \phi_{x x}+3 u_{j-2, x} \phi_{x}^{2}\right]\right. \\
& \left.\quad+(\mathrm{J}-5)\left[u_{j-3} \phi_{x x x}+3 u_{j-3, x} \phi_{x x}+3 u_{j-3, x x} \phi_{x}\right]+u_{j-4, x x x}\right\} \\
& +\sigma\left\{(j-5)\left[(j-4) u_{j-2} \phi_{y}^{2}+u_{j-3} \phi_{y y}+2 u_{j-3, y} \phi_{y}\right]+u_{j-4, y y}\right\}=0
\end{aligned}
$$

where coefficients $\boldsymbol{u}_{\boldsymbol{j}}$ with negative $\mathrm{j}$ are taken to be zero. For $\mathbf{j}=0$ we obtain

$$
u_{0}=-12 \mu \phi_{x}^{2}
$$

Using (4) in (3) collecting coefficients of $u_{j}$ we obtain

$$
(j+1)(j-4)(j-5)(j-6) u_{j}=h_{j}\left(\phi_{x}, \phi_{y}, \phi_{t}, \ldots, u_{0}, \ldots, u_{j-1}\right)
$$

where $h j$ is a non-linear function. We can see that $j=-1,4,5,6$, are resonances at which $U j$ becomes arbitrary. Resonance at -1 corresponds to the arbitrariness of $\phi$. 
For the system to have Painleve property (PP) there should be 3 more arbitrary coefficients $u_{4}, u_{5}$, and $u_{6}$ occurring at $7=4,5$, and 6 respectively without any constraint on 0 . That is, at those values the compatibility relation $h j-0$, should be consistently satisfied. When $h j \neq 0$ at a resonance value $j$, we can make $u_{j}$ arbitrary by including logarithmic terms in the series or there will be a constraint on 0 at that 7 . In that case the arbitrariness of 0 is lost and we may say that the equation has conditional PP. Using the constraints we solve for the particular form of 0 and it can be used to find special solutions of such conditional PP systems.

For successive values of 7 , from the recurrence relation (3) we obtain

$$
\begin{aligned}
& j=1 \quad u_{1}=\frac{12\left(-v \phi_{x}+5 \mu \phi_{x x}\right)}{5}, \\
& j=2 \quad u_{2}=\frac{v^{2}}{25 \mu}-\frac{\sigma \phi_{y}^{2}}{\phi_{x}^{2}}-\frac{\phi_{t}}{\phi_{x}}+\frac{6 v \phi_{x x}}{5 \phi_{x}}+\frac{3 \mu \phi_{x x}^{2}}{\phi}-\frac{4 \mu \phi_{x x x}}{\phi_{x}}, \\
& \mathbf{i}-\hat{\mathbf{J}} \quad u_{3}-{ }_{\psi_{x}^{2}}^{\sigma \phi_{v v}}-{ }_{1\lrcorner \nu \mu^{2} \psi_{x}}^{v^{3}}+\begin{array}{ccc}
\phi_{x t} & \sigma \phi_{v}^{2} \phi_{x x} & \phi_{t} \phi_{x x} \\
\dot{\psi}_{x}^{2} & \phi_{x}^{4} & \phi_{x}^{\mathbf{3}}
\end{array} ; \frac{3 v \phi_{x x}^{2}}{5 \phi_{x}^{3}} \\
& +\frac{3 \mu \phi_{x x}^{3}}{\phi_{x}^{4}}-\frac{2 v \phi_{x x x}}{5 \phi_{x}^{2}}-\frac{4 \mu \phi_{x x} \phi_{x x x}}{\phi_{x}^{3}}+\frac{\mu \phi_{x x x x}}{\phi_{x}^{2}} . \\
& 7=4 \quad u_{4} \text { is arbitrary with the constraint, } \\
& 0=\phi_{y y} \phi_{x}^{2}-2 \phi_{y} \phi_{x} \phi_{x y}+\phi_{y}^{2} \phi_{x x} \equiv A \text {. } \\
& 7=5 \quad u_{5} \text { is arbitrary with } \\
& \mathbf{o}=2 \phi_{x}^{2} \phi_{x}^{2}-\phi_{x}^{3} \phi-4 \phi_{y} \phi_{x} \phi_{x y} \phi_{x x} \\
& +2 \phi_{v}^{2} \phi_{x x}^{2}+2 \phi_{y} \phi_{x}^{2} \phi_{x x y}-\phi_{v}^{2} \phi_{x} \phi_{x x x} \equiv B .
\end{aligned}
$$

We can see that (10) is satisfied identically if (9) is satisfied, because $B / \phi_{x}^{3}=\left(A / \phi_{x}^{2}\right)_{x}$. There is incompatibility at $j=6$, and the recurrence relation is too lengthy and complicated. $h_{6}$ does not vanish identically, when $u_{4}$ and $u_{5}$ are arbitrary. From this analysis we see that $2 \mathrm{~d}-\mathrm{KdVB}$ is non-Painleve and because of Painleve conjecture it is non-integrable.

\section{Exact solutions}

To find special solutions we can truncate the Painleve expansion (2) at a particular order and find constraining equations on 0 . Solving for 0 and substituting in (2) we obtain the corresponding special solution for system (1). The solution we obtain after this truncation procedure is not the general solution of the system because they do not contain sufficient number of arbitrary coefficients, but they are exact. In the truncation procedure we impose the condition that coefficients of the higher order terms in the expansion are zero.

Case (i). Let us start with $u_{j}-0$ for all $7 \geqslant 1$, so that the solution to the equation has the form

$$
u=u_{0} \phi^{-2}
$$




\section{P Joy}

Using the recurrence relations (3), we obtain

$$
\begin{aligned}
0= & 5 \mu \phi_{x x}-v \phi_{x} . \\
0= & -\sigma \phi_{y}^{2}-\phi_{t} \phi_{x}+9 v \phi_{x} \phi_{x x}-27 \mu \phi_{x x}^{2}-12 \mu \phi_{x} \phi_{x x x} . \\
0= & \sigma \phi_{y y} \phi_{x}^{2}+3 \phi_{x}^{2} \phi_{x t}+4 \sigma \phi_{y} \phi_{x} \phi_{x y}+2 \phi_{t} \phi_{x} \phi_{x x}-12 v \phi_{x} \phi_{x x}^{2} \\
& +12 \mu \phi_{x x}^{3}-7 v \phi_{x}^{2} \phi_{x x x}+44 \mu \phi_{x} \phi_{x x} \phi_{x x x}+9 \mu \phi_{x}^{2} \phi_{x x x x} . \\
0= & -\sigma \phi_{x y}^{2}-\sigma \phi_{x} \phi_{x y y}-\phi_{x t} \phi_{x x}-\phi_{x} \phi_{x x t}+3 v \phi_{x x} \phi_{x x x} \\
& -3 \mu \phi_{x x x}^{2}+v \phi_{x} \phi_{x x x x}-4 \mu \phi_{x x} \phi_{x x x x}-\mu \phi_{x} \phi_{x x x x x} .
\end{aligned}
$$

A solution for $\phi$ of the form

$$
(j)=\exp \left(\xi-\xi_{0}\right)+A,
$$

where $\xi_{0}$, and $A$ are arbitrary and

$$
\xi=k x+l y-\omega t
$$

exists for the above equations (12-15). Here,

$$
k=\frac{\mathrm{v}}{5 \mu},(1)=-\frac{6 v^{3}}{125 \mu^{2}}+\frac{5 \sigma l^{2} \mu}{\mathrm{v}}
$$

and $l$ is arbitrary. It is to be noted here that (16) is not the most general solution of equations (12-15), it is the simplest non-trivial solution one can obtain for $\phi$. If we could find other solutions for $\phi$ we can use them in (11) to find other solutions of the original system (1). Here we did not assume any particular form for solutions of the original system.

With this, the solution $u$ given by (11) becomes

$$
u=\frac{-12 v^{2}}{25 \mu} \frac{1}{\left(1+A \exp \left[-\left(\xi-\xi_{0}\right)\right]\right)^{2}} .
$$

When $A=0$, the trivial solution $u=$ constant is obtained. When $A=1$ we get one of the solutions given in [10]: Then (18) can be written as

$$
u=\frac{3 v^{2}}{25 \mu}\left[S^{2}-2 T-2\right]
$$

where

and

$$
S=\operatorname{sech}\left[\frac{1}{2}\left(\xi-\xi_{0}\right)\right]
$$

$$
\mathrm{T}=\tanh \left[\frac{1}{2}\left(\xi-\xi_{0}\right)\right] \text {. }
$$

Case (ii). Let us truncate the solution at the next order, i.e. $u_{j^{-}} 0$ for all $j \geqslant 2$, $\left(u_{0} \neq 0, u_{1} \neq 0\right)$. Then

$$
u=\frac{u_{0}}{\phi^{2}}+\stackrel{u}{\psi}
$$




\section{Exact solutions of $2 d-K d V B$}

From recurrence relations, we have

$$
\begin{aligned}
& 0=\frac{v^{2}}{25 \mu}-\frac{\sigma \phi_{y}^{2}}{\phi_{x}^{2}}-\frac{\phi_{t}}{\phi_{x}}+\frac{6 v \phi_{x x}}{5 \phi_{x}}+\frac{3 \mu \phi_{x x}^{2}}{\phi_{x}^{2}}-\frac{4 \mu \phi_{x x x}}{\phi_{x}} . \\
& 0=\frac{-v \sigma \phi_{y}^{2} \phi_{x}}{\zeta_{\ddot{A}}}-\frac{v \phi_{t} \phi_{x}^{2}}{5 \mu}+\sigma \phi_{y j} \phi_{r_{0}}^{2}+3 \phi_{r}^{2} \phi_{z}+4 \sigma \phi_{y} \phi_{z} \phi_{x y}+\sigma \phi_{y}^{2} \phi_{x x} \\
& +3 \phi \phi_{x} \phi_{\overline{x x}} \frac{18 v \phi \phi_{x}^{2}}{\jmath}-3 \mu \Phi_{x x}^{s}-\frac{18 v \phi_{x}^{2} \phi_{x x x}}{\jmath}-9 \hat{\mu} \phi_{x}^{<} \phi_{x x x x} . \\
& \mathbf{0}=\frac{v \pi \phi_{y y} \phi_{x}}{5}+\frac{2 v \phi_{x} \phi_{x t}}{5}+\frac{2 v \sigma \phi_{y} \phi_{x y}}{5}-2 \mu \sigma \phi_{x y}^{2}-2 \mu \sigma \phi_{x} \phi_{x y y} \\
& +\frac{v \phi_{t} \phi_{x x}}{5}-\mu \sigma \phi_{y y} \phi_{x x}-3 \mu \phi_{x t} \phi_{x x}-\frac{3 v^{2} \phi_{x x}^{2}}{25}-3 \mu \phi_{x} \phi_{x x t} \\
& -2 \mu \sigma \phi_{y} \phi_{x x y}-\mu \phi_{t} \phi_{x x x}-\frac{8 v^{2} \phi_{x} \phi_{x x x}}{25}+\frac{24 \mu \nu \phi_{x x} \phi_{x x x}}{5} \\
& +2 \mu^{2} \phi_{x x x}^{2}+\frac{18 \mu v \phi_{x} \phi_{x x x x}}{5}-3 \mu^{2} \phi_{x x} \phi_{x x x x}-6 \mu^{2} \phi_{x} \phi_{x x x x x} . \\
& 0=-v \sigma \phi_{x y y}-v \phi_{x x t}+5 \mu \sigma \phi_{x x y y}+5 \mu \phi_{x x x t}+v^{2} \phi_{x x x x} \\
& -6 \mu v \phi_{x x x x x}+5 \mu^{2} \phi_{x x x x x x x} \text {. }
\end{aligned}
$$

These equations satisfy a solution for $\phi$ of the form (16) with

$$
k=+\frac{\mathbf{v}}{5 \mu}, \mathrm{CO}=\left(-\frac{6 v^{3}}{1 \bar{\Sigma} J \mu^{2}} \pm \frac{5 \sigma l^{2} \mu}{v}\right)
$$

and $l$ arbitrary. Here we get two waves in opposite directions. In this case, the solution (22) will be (after substituting for $\boldsymbol{u}_{0}, \boldsymbol{u}_{1}$ )

$$
u=-12 v^{2}\left\{\frac{1}{25 \mu}\left\{\frac{-1+1}{\left(1+A \exp \left[-\left(\xi-\xi_{0}\right)\right]\right)^{2}}+\frac{1}{1+A \exp \left[-\left(\xi-\xi_{0}\right)\right]}\right\} .\right.
$$

When $A=1$ we obtain the solution given by (12) in [9] and (15) in [10],

$$
u=\frac{3 v^{2}}{25 \mu}\left[S^{2} \mp 2 T \mp 2\right],
$$

where $\mathrm{S}$ and $T$ are as defined in (20) and (21).

Case (iii). Now we set $u_{j}=0$ for all $j \geqslant 3\left(u_{0}, u_{1}, u_{2} \neq 0\right)$. Then

$$
u=\frac{u_{0}}{\phi^{2}}+\frac{u_{1}}{\phi}+u_{2} \text {. }
$$

Now the recurrence relations give

$$
0=\frac{\sigma \phi_{y y}}{\phi_{x}}-\frac{v^{3}}{125 \mu^{2}}+\frac{\phi_{x t}}{\phi_{x}}-\frac{\sigma \phi_{y}^{2} \phi_{x x}}{\phi_{x}^{3}}-\frac{\phi_{t} \phi_{x x}}{\phi_{x}^{2}}+\frac{3 v \phi_{x x}^{2}}{5 \phi_{x}^{2}}
$$


M P Joy

$$
\begin{aligned}
& +\frac{3 \mu \phi_{x x}^{3}}{\phi_{x}^{3}}-\frac{2 v \phi_{x x x}}{5 \phi_{x}}-\frac{4 \mu \phi_{x x} \phi_{x x x}}{\phi_{x}^{2}}+\frac{\mu \phi_{x x x x}}{\phi_{x}} \\
& 0=\frac{v \sigma \phi_{y y} \phi_{x}}{5 \mu}-\frac{2 v \sigma \phi_{y} \phi_{x y}}{5 \mu}-2 \sigma \phi_{x} \phi_{x y y}-\sigma \phi_{y y} \phi_{x x}+\frac{v \sigma \phi_{y}^{2} \phi_{x x}}{5 \mu \phi_{x}}+\frac{3 v^{3} \phi_{x} \phi_{x x}}{125 \mu^{2}} \\
& +\phi_{x t} \phi_{x x}+\frac{4 \sigma \phi_{y} \phi_{x y} \phi_{x x}}{\phi_{x}}-\frac{3 \sigma \phi_{y}^{2} \phi_{x x}^{2}}{\phi_{x}^{2}}-\frac{\phi_{t} \phi_{x x}^{2}}{\phi_{x x}}+\frac{3 v \phi_{x x}^{3}}{5 \phi_{x}}+\frac{9 \mu \phi_{x x}^{4}}{\phi_{x}^{2}} \\
& -2 \phi_{x} \phi_{x x t}+2 \phi_{t} \phi_{x x x}+\frac{2 \sigma \phi_{y}^{2} \phi_{x x x}}{\phi_{x}}-2 v \phi_{x x} \phi_{x x x}-\frac{22 \mu \phi_{x x}^{2} \phi_{x x x}}{\phi_{x}} \\
& +8 \mu \phi_{x x x}^{2}+\frac{4 v \phi_{x} \phi_{x x x x}}{5}+7 \mu \phi_{x x} \phi_{x x x x}-2 \mu \phi_{x} \phi_{x x x x x}
\end{aligned}
$$

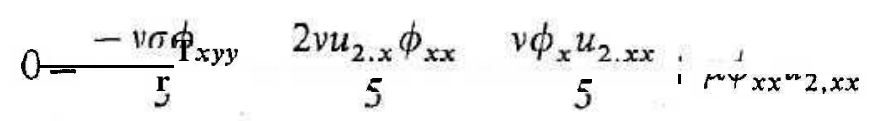

$$
\begin{aligned}
& -\frac{v \phi_{x x t}}{5}+\mu \sigma \phi_{x x y y}-\frac{v u_{2} \phi_{x x x}}{5}+2 \mu u_{2, x} \phi_{x x x}+\mu \phi_{x x x t} \\
& +\frac{v^{2} \phi_{x x x x}}{5}+\mu u_{2} \phi_{x x x x}-\frac{6 \mu \nu \phi_{x x x x x}}{5}+\mu^{2} \phi_{x x x x x x} . \\
& 0=\operatorname{ffU}_{2, "}+\bar{u}_{\overline{2}, x}+u_{2, x t}+" 2 u_{2, x x}-v u_{2, x x x}+\mu u_{2, x x x x} .
\end{aligned}
$$

Here we see that at $j=6$ the recurrence relation gives $2 \mathrm{~d}-\mathrm{KdVB}$ for $u_{2}$. Hence (29) may be considered as an auto-Bäcklund transformation. We can find a solution for $\phi$ from (30-33) in the form (16) with

$$
k= \pm \frac{\mathrm{v}}{5 \mu}, \omega=\frac{v c}{5 \mu} \pm \frac{5 \sigma l^{2} \mu}{v}
$$

and $l$ arbitrary, where,

$$
c=\frac{\omega k-\sigma l^{2}}{k^{2}}=-\frac{6 v^{2}}{25 \mu} \pm \lambda .
$$

Here we note that $X$ is the value of $u_{2}$ which can be an arbitrary non-zero constant. Now the solution for (1), given by (29) is

$$
u=\frac{-12 v^{2}}{25 \mu}\left\{\frac{\searrow}{\left(1+A \exp \left[-\left(\xi-\xi_{0}\right)\right]\right)^{2}}+\frac{-1 \pm 1}{1+A \exp \left[-\left(\zeta-\xi_{0}\right)\right]}\right\}+\lambda .
$$

For $A=1$ we could obtain (17) of [10] or (10) of [8].

$$
u=\frac{3 v^{2}}{25 \mu}\left[S^{2} \mp 2 T\right] \pm \mathrm{c},
$$

where $S$ and $T$ are defined as in (20) and (21). 


\section{Exact solutions of $2 d-K d V B$}

If (29) is considered as a Bäcklund transformation, travelling wave solutions differing by a constant is only obtained. Therefore we do not obtain non-trivially new travelling wave solutions from the known solutions using (29).

The solution (36) can be obtained from (28) by using the fact that (1) is invariant under the transformation [10]

$$
u^{*}=u+\lambda, \quad \mathrm{x}^{*}=x+\lambda t, \quad t^{*}=t, \quad y^{*}=y .
$$

The solution (36) of $2 \mathrm{~d}-\mathrm{KdVB}$ can be written in terms of travelling wave solutions of KP and $2 \mathrm{~d}$-Burgers equations [9]. If we continue the process of truncation to higher order we do not obtain any new solutions. From (3) we see that at $j=6,7$ and 8 compatibility conditions require $u_{3}=0$ and hence all other higher order coefficients should be zero forcompatibility.

\section{Conclusion}

We used the Painleve test as described by Weiss et al [1] to study the integrability of $2 \mathrm{~d}$ Korteweg-de Vries-Burgers equation and showed that it is non-integrable. We obtained exact travelling wave solutions by using the method of truncation of Painleve series at successive orders and all previously known travelling wave solutions as special cases of our solution to the system. Moreover we may obtain other types of exact solutions, if we can solve the constraining equations on $\phi$ at each order. Here we did not assume any particular form for the solutions unlike others $[8,9,10]$, where they assumed the travelling wave form for solutions and searched for them. In such methods we obtain only special solutions of the assumed form, if they exist. The method of P-analysis is algorithmic and it gives details on the integrability aspects of the equation also.

\section{Acknowledgement}

The author acknowledges the financial support from National Board for Higher Mathematics, Department of Atomic Energy, India.

\section{References}

[1] J Weiss, M Tabor and G Carnevale, The Painleve property for partial differential equations, J. Math. Phys. 24, 522-526 (1983)

[2] R Conte, Invariant Painleve analysis of partial differential equations, Phys. Lett. A140, 383-390 (1989)

[3] A Ramani, B Grammaticos and T Bountis, The Painleve property and singularity analysis of integrable and non-integrable systems, Phys. Rep. 180, 159-245(1989)

[4] F Cariello and M Tabor, Painleve expansions for non-integrable evolution equations, Physica D39, 77-94 (1989)

[5] R S Johnson, A non-linear equation incorporating damping and dispersion, J. Fluid Mech. 42, 49-60 (1970)

[6] L van Wijngaarden, One-dimensional flow of liquids containing small gas bubbles, Ann. Rev. Fluid Mech. 4, 369-395 (1972)

[7] P Barrera and T Brugarino, Similarity solutions of the generalized Kadomtsev-PetviashviliBurgers equation, Nuovo Cimento B92, 142-156(1986)

[8] W Ma, An exact solution to two-dimensional Korteweg-de Vries-Burgers equation, J. Phys. A26, L17-L20 (1993) 


\section{P Joy}

[9] Li Zhibin and Wang Mingliang, Travelling wave solutions to two-dimensional KdVBurgers equation, J. Phys. A26, 6027-6031 (1993)

[10] E J Parkes, Exact solutions to the two dimensional Korteweg-de Vries-Burgers equation, J. Phys. A27, L497-L501 (1994)

[11] W D Halford and M Vlieg-Hulstman, Korteweg-de Vries-Burgers equation and the Painlevé property, J. Phys. A25, 2375-2379 (1992) 